\title{
Stereoselective Synthesis of a MCHr1 Antagonist
}

Denise Andersen,${ }^{* \dagger}$ Thomas Storz,${ }^{\dagger}$ Pingli Liu ${ }^{\Pi}$, Xin Wang, ${ }^{\dagger}$ Leping Li ${ }^{*}$ Pingchen Fan, ${ }^{*}$ Xiaoqi Chen, ${ }^{\ddagger}$ Alan Allgeier, ${ }^{\dagger}$ Alain Burgos, ${ }^{\S}$ Jason Tedrow, ${ }^{\dagger}$ Jean Baum, ${ }^{\dagger}$ Ying Chen ${ }^{\dagger}$ Rich Crockett ${ }^{\dagger}$ Liang Huang, ${ }^{\dagger}$ Rashid Syed,${ }^{\dagger}$ Robert D. Larsen ${ }^{\dagger}$ and Mike Martinelli ${ }^{\dagger}$

${ }^{\dagger}$ Chemistry Process Research and Development, Amgen Inc.

Thousand Oaks, California, Medicinal Chemistry, ${ }^{*}$ Amgen SF, South San Francisco, California, Process Chemistry, ${ }^{\Pi}$ Incycte Corporation, Wilmington, Delaware and ${ }^{\S}$ Pharmaceutical Products PPG-Sipsy, Z.I. La Croix Cadeau B. P. 79, F-49242 Avrillé Cedex, France danderse@amgen.com

\section{Supporting Information}

\section{Table of Contents}

Section A: General Information

Section B: ${ }^{1} \mathrm{H}-\mathrm{NMR}$ and ${ }^{13} \mathrm{C}-\mathrm{NMR}$ Spectra

Section C: ORTEP $(R)$-2-Chloromandelate salt of 2
S-2

S-3

S-27 


\section{Section A: General Information}

NMR spectra were recorded in the specified solvent using a $300 \mathrm{MHz}, 400 \mathrm{MHz}$ or 600 MHz spectrometer. IR spectra were recorded using a universal ATR sampling unit and melting points were obtained either using a capillary melting point apparatus or by differential scanning calorimetry (DSC). 


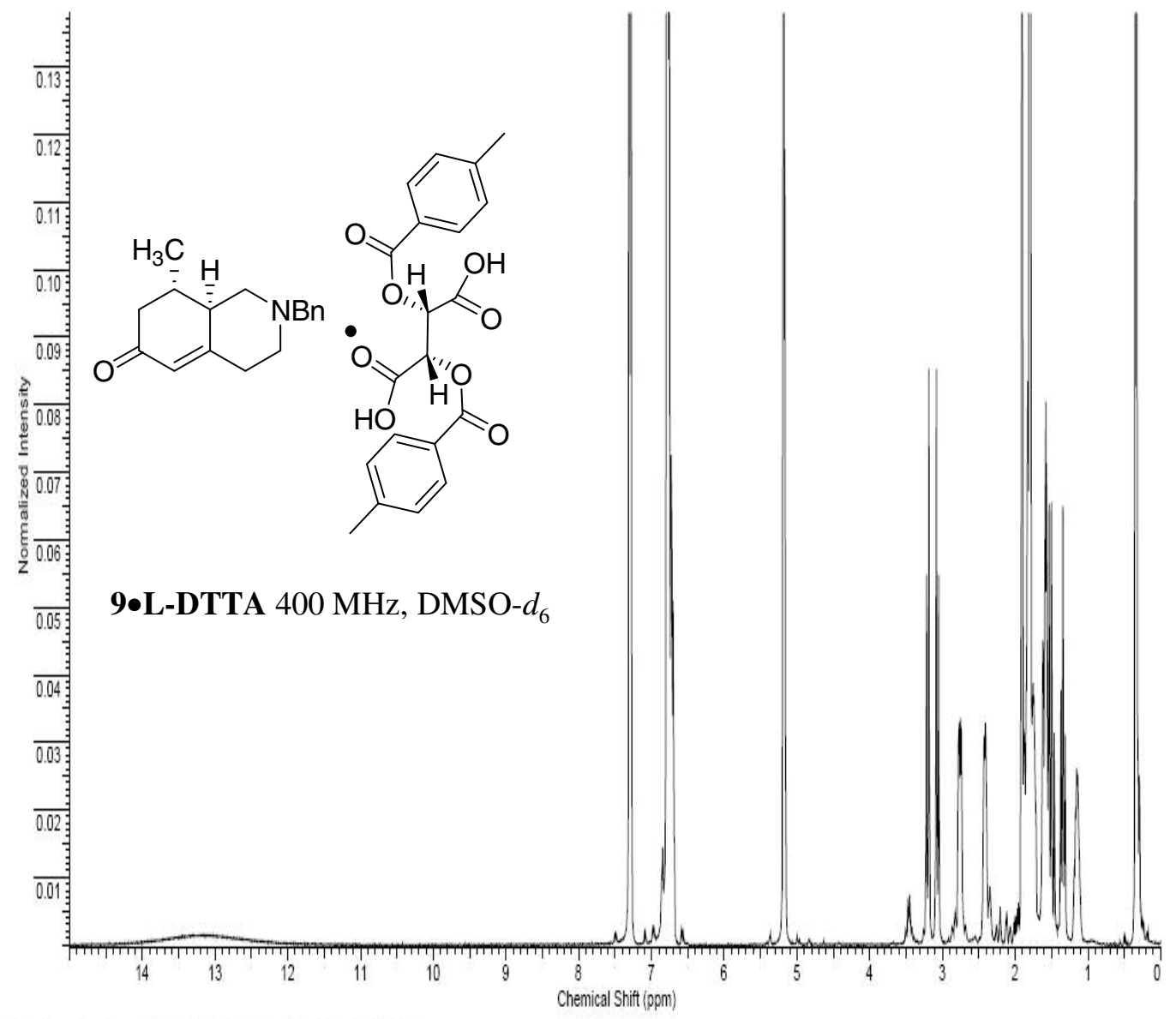

\begin{tabular}{|c|c|c|c|c|}
\hline No. & $($ ppm) & Value & Absolute Value & Non-Negative Value \\
\hline 1 & {$[0.23 \ldots 0.41]$} & 3.000 & $1.69641 \mathrm{e}+9$ & 3.000 \\
\hline 2 & {$[1.07 \ldots 1.24]$} & 0.913 & $5.16502 \mathrm{e}+8$ & 0.913 \\
\hline 3 & {$[1.27 . .1 .41]$} & 0.933 & $5.27718 \mathrm{e}+8$ & 0.933 \\
\hline 4 & {$[1.41 \ldots 1.65]$} & 3.107 & $1.75709 \mathrm{e}+9$ & 3.107 \\
\hline 5 & {$[1.68 \ldots 1.87]$} & 8.454 & $4.78026 \mathrm{e}+9$ & 8.454 \\
\hline 6 & {$[1.88 \ldots 1.96]$} & 1.948 & $1.10163 \mathrm{e}+9$ & 1.948 \\
\hline 7 & {$[2.31 . .2 .46]$} & 1.037 & $5.86673 \mathrm{e}+8$ & 1.037 \\
\hline 8 & {$[2.66 .2 .85]$} & 1.044 & $5.90601 \mathrm{e}+8$ & 1.044 \\
\hline 9 & {$[2.98 \ldots 3.14]$} & 0.903 & $5.10533 \mathrm{e}+8$ & 0.903 \\
\hline 10 & {$[3.14 \ldots 3.27]$} & 0.906 & $5.12350 \mathrm{e}+8$ & 0.906 \\
\hline 11 & {$[4.92 .5 .38]$} & 2.867 & $1.62139 \mathrm{e}+9$ & 2.867 \\
\hline 12 & {$[6.62 .6 .97]$} & 8.920 & $5.04392 \mathrm{e}+9$ & 8.920 \\
\hline 13 & {$[7.15 . .7 .47]$} & 3.931 & $2.22296 \mathrm{e}+9$ & 3.931 \\
\hline 14 & {$[12.07 . .14 .63]$} & 0.893 & $5.05027 \mathrm{e}+8$ & 0.893 \\
\hline
\end{tabular}




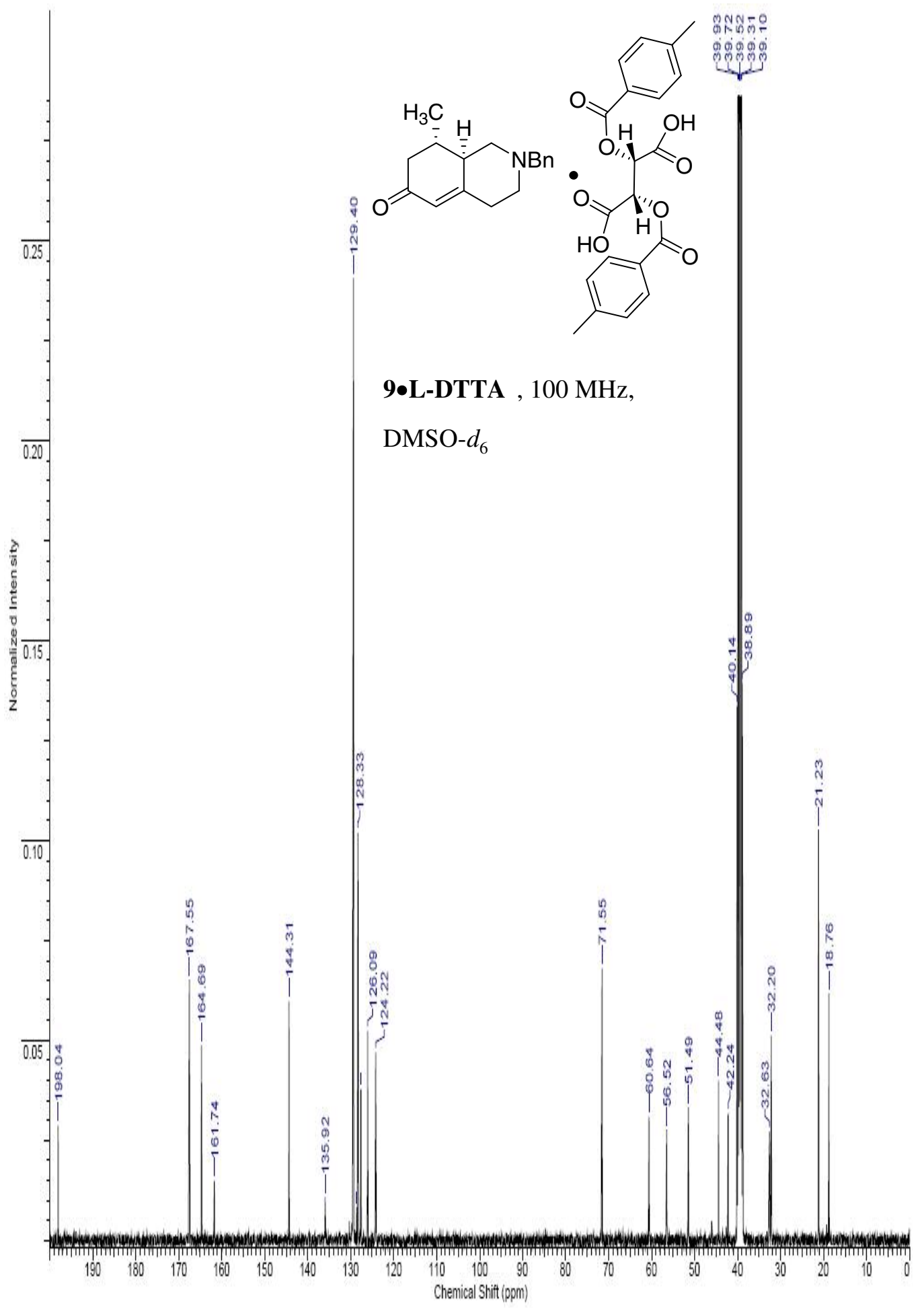




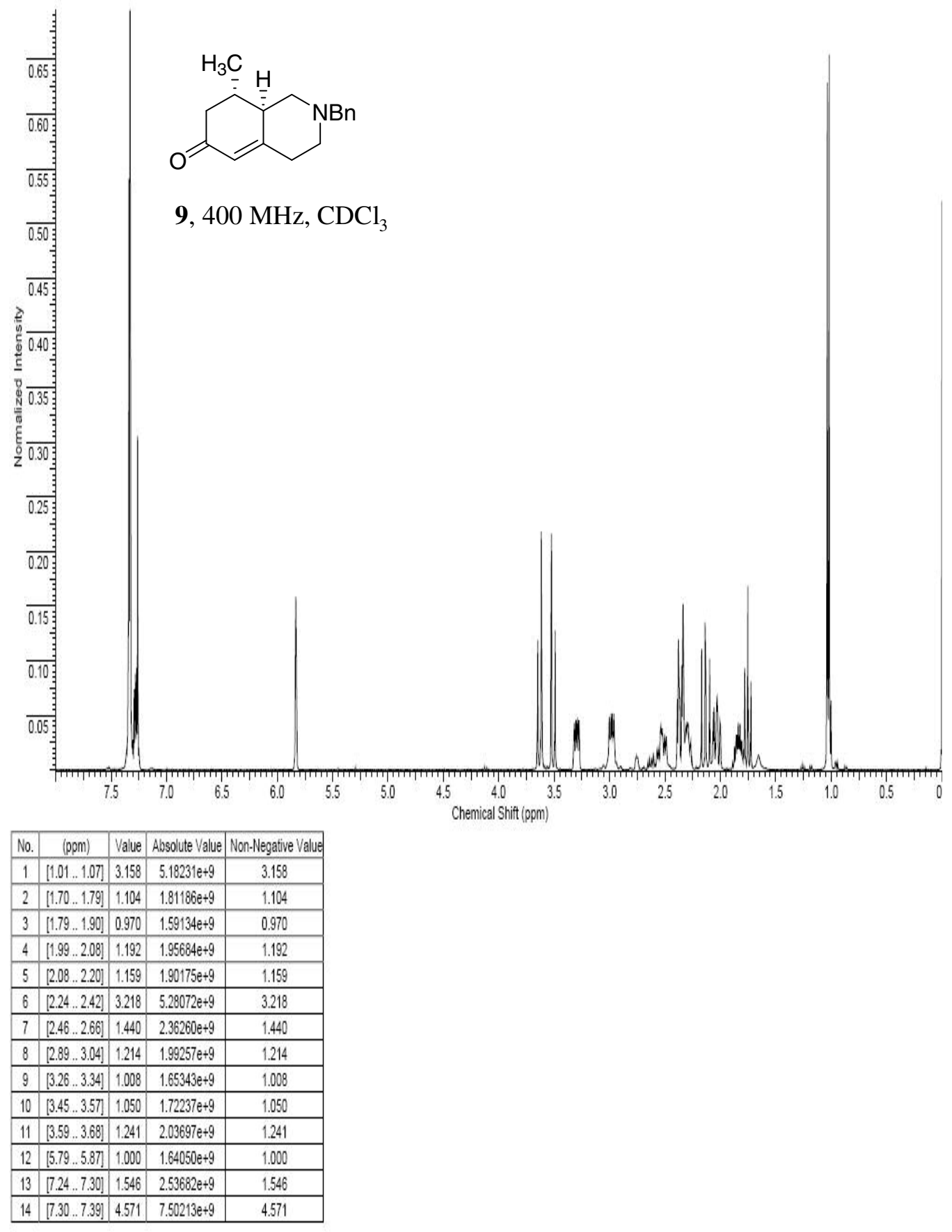




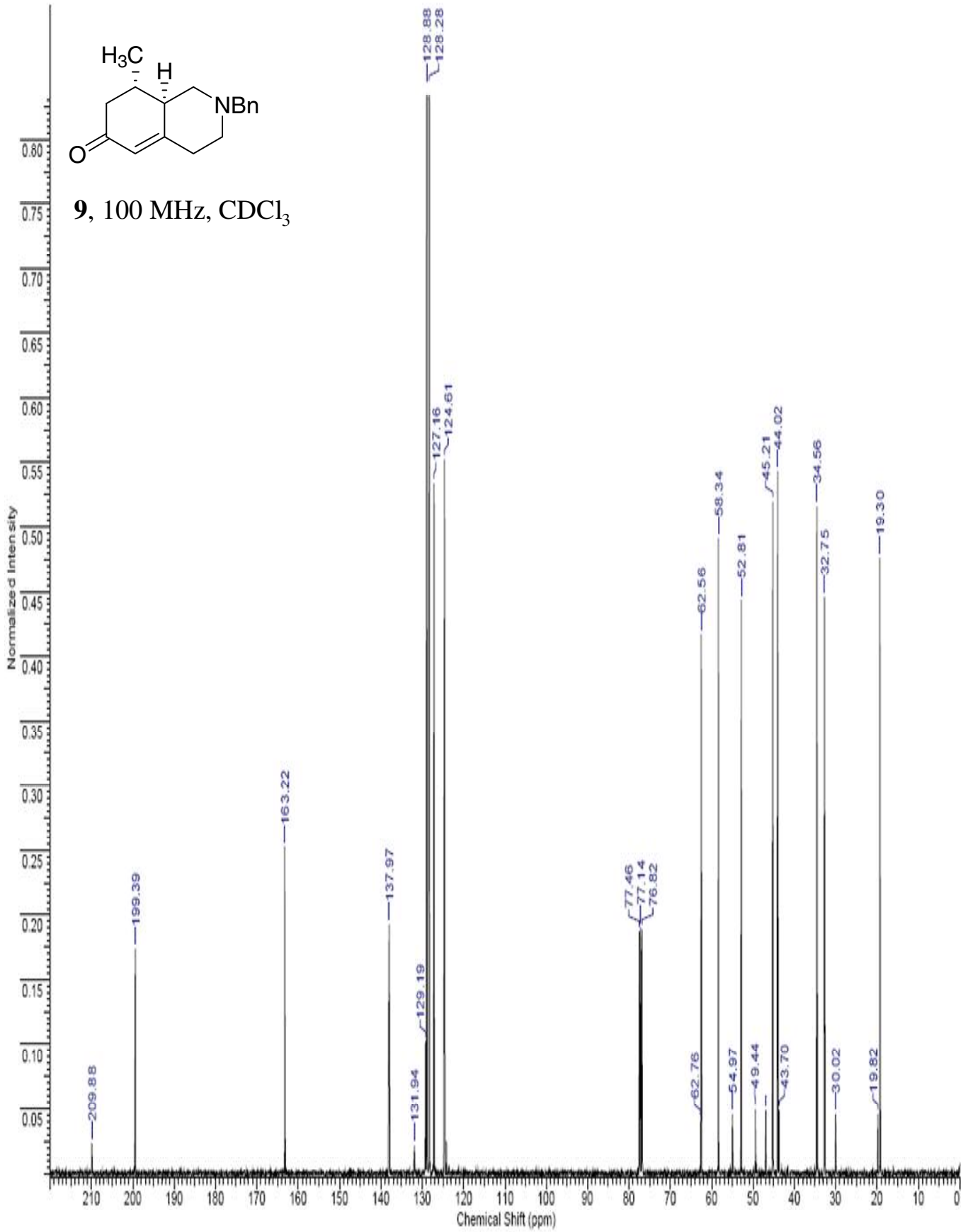

S-6 


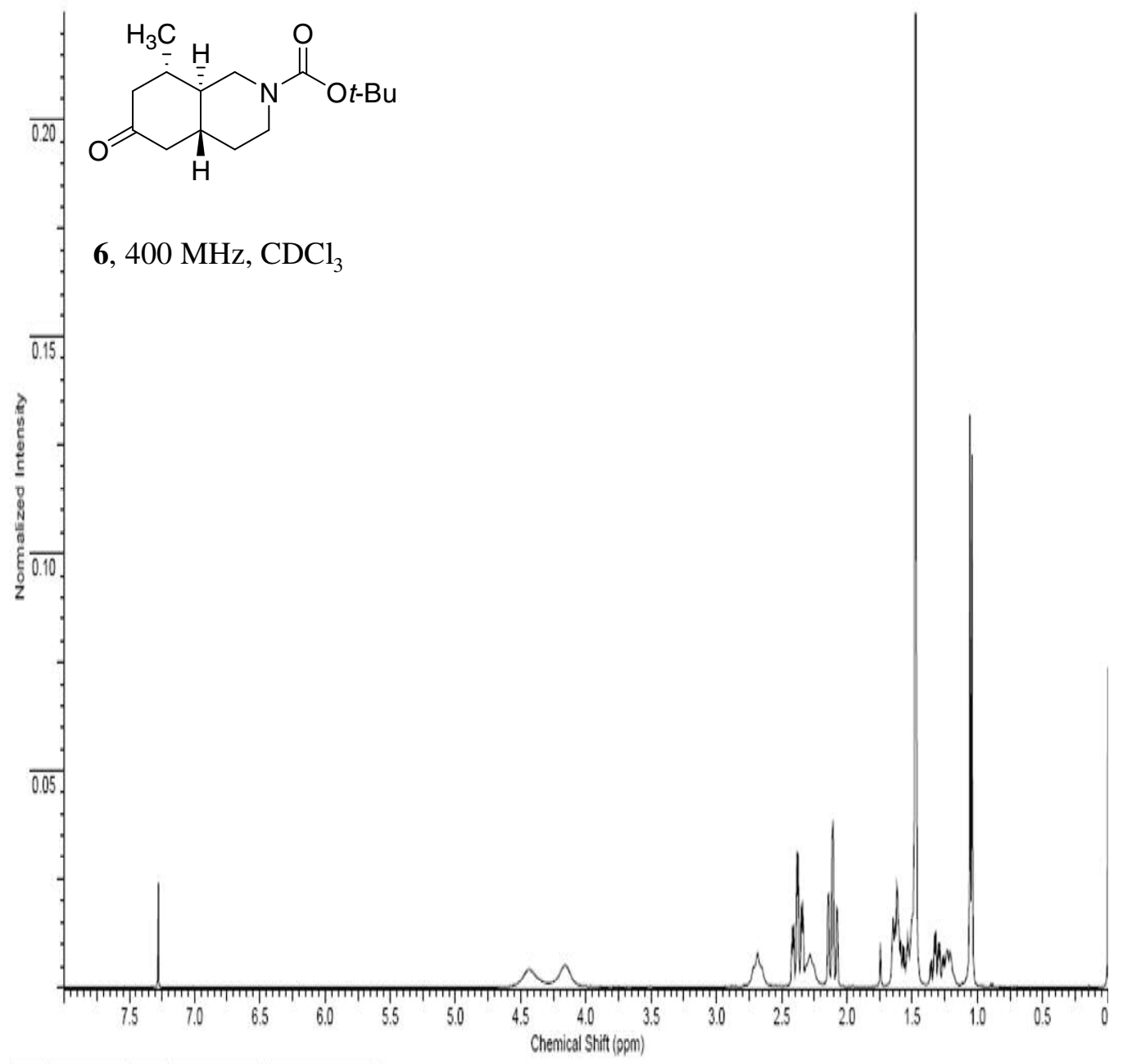

\begin{tabular}{|c|c|c|c|c|}
\hline No. & $($ pom $)$ & Value & Absolute Value & Non-Negative Value \\
\hline 1 & {$[1.00 \ldots 1.10]$} & 3.000 & $6.24054 \mathrm{e}+8$ & 3.000 \\
\hline 2 & {$[1.14 \ldots 1.40]$} & 2.084 & $4.33462 \mathrm{e}+8$ & 2.084 \\
\hline 3 & {$[1.41 \ldots 1.51]$} & 9.420 & $1.95963 \mathrm{e}+9$ & 9.420 \\
\hline 4 & {$[1.51 \ldots 1.69]$} & 2.766 & $5.75288 \mathrm{e}+8$ & 2.766 \\
\hline 5 & {$[2.04 \ldots 2.18]$} & 2.032 & $4.22730 \mathrm{e}+8$ & 2.032 \\
\hline 6 & {$[2.19 \ldots 2.33]$} & 0.909 & $1.89119 \mathrm{e}+8$ & 0.909 \\
\hline 7 & {$[2.33 \ldots 2.45]$} & 2.108 & $4.38487 \mathrm{e}+8$ & 2.108 \\
\hline 8 & {$[2.52 \ldots 2.87]$} & 1.050 & $2.18499 \mathrm{e}+8$ & 1.050 \\
\hline 8 & {$[4.04 \ldots 4.27]$} & 0.979 & $2.03751 \mathrm{e}+8$ & 0.979 \\
\hline 10 & {$[4.27 \ldots 4.63]$} & 1.015 & $2.11090 \mathrm{e}+8$ & 1.015 \\
\hline
\end{tabular}




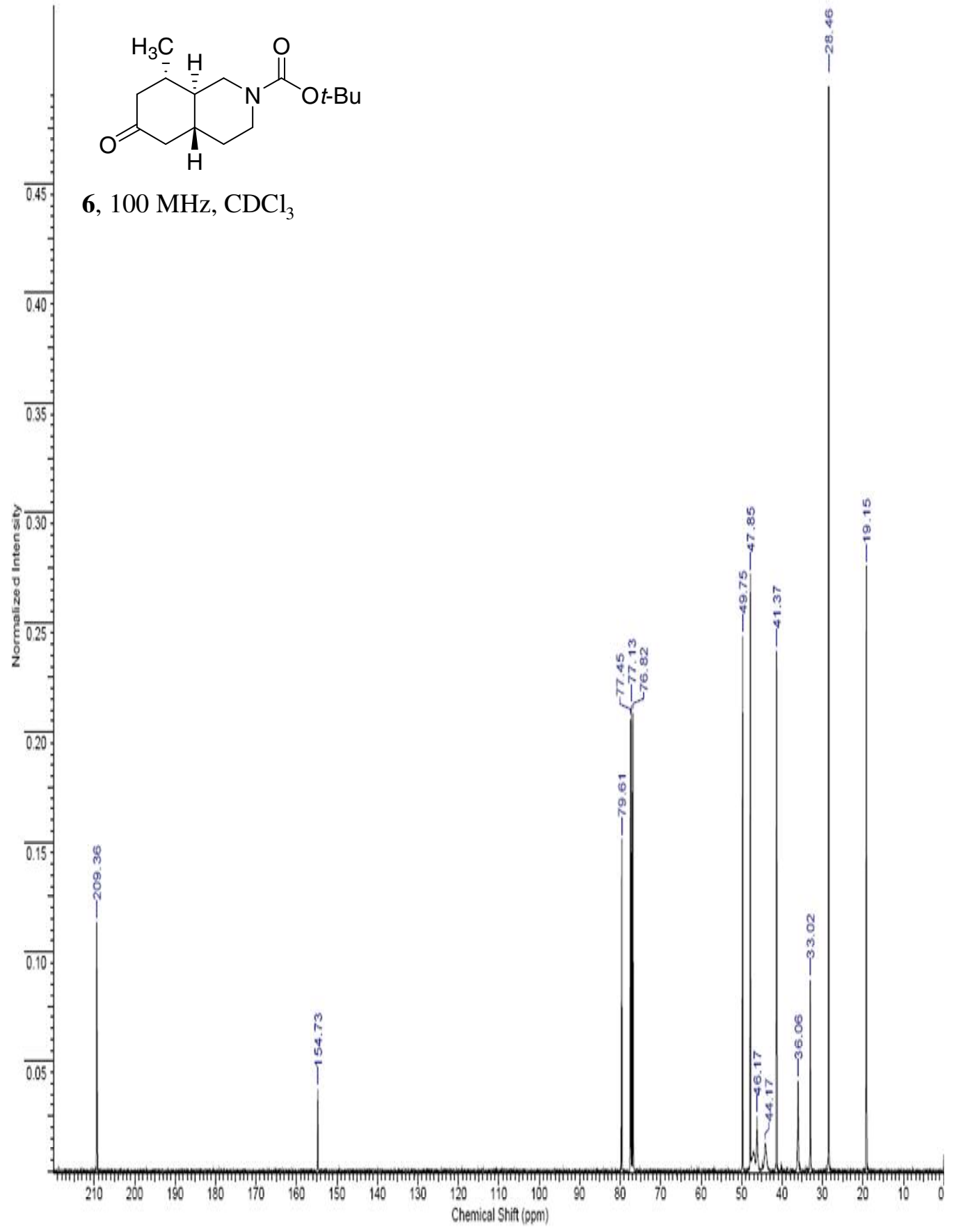




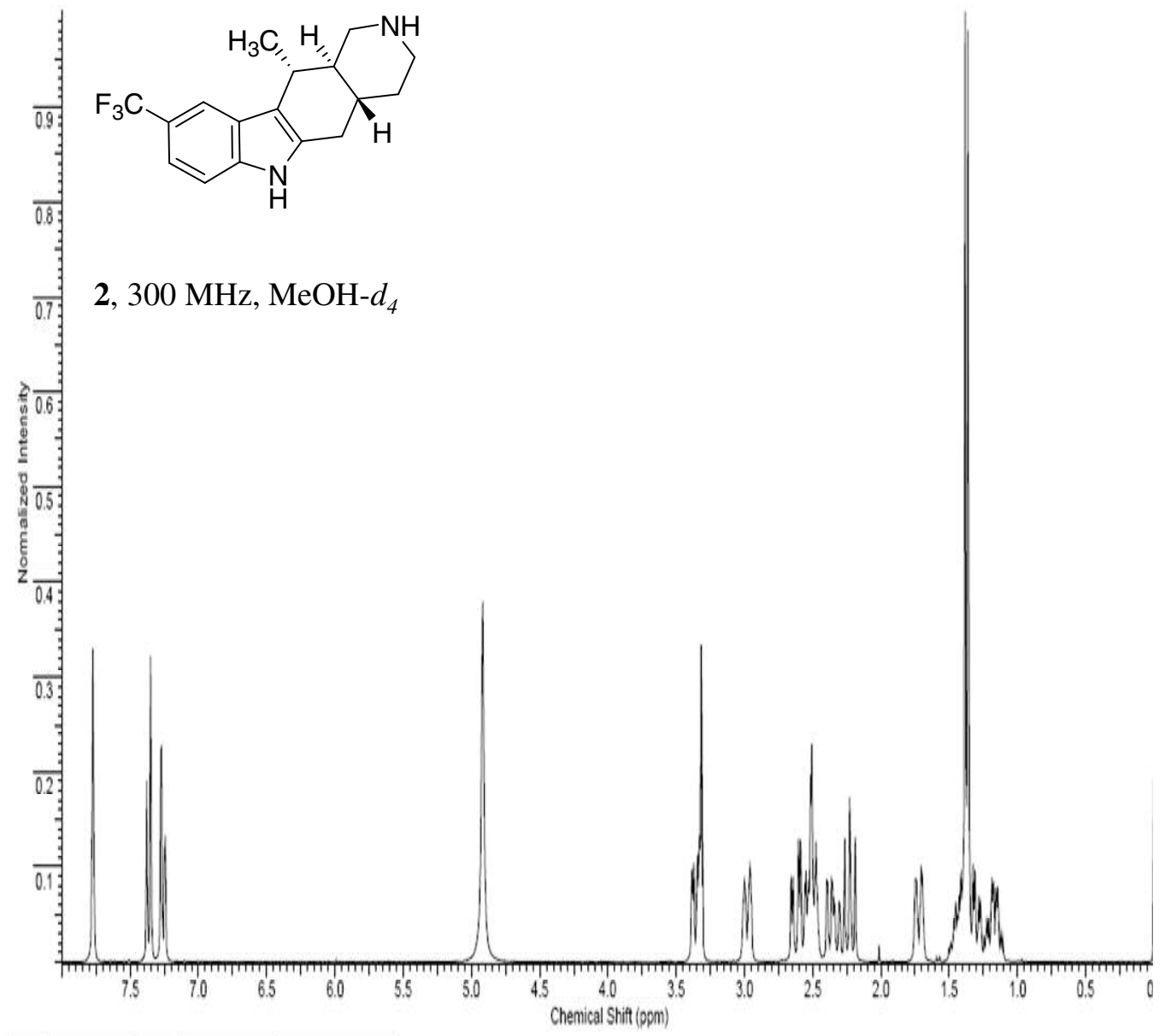

\begin{tabular}{|c|c|c|c|c|}
\hline No. & (ppm) & Value & Absolute Value & Non-Negative Value \\
\hline 1 & {$[1.07,1.34]$} & 1.930 & $8.89820 e+9$ & 1.930 \\
\hline 2 & {$[1.34,1,400]$} & 3.327 & $1.53364 \mathrm{e}+10$ & 3.327 \\
\hline 3 & {$[1,40,1,52]$} & 0.899 & $4.142366+9$ & 0.899 \\
\hline 4 & {$[1,62 \ldots 1,1,78]$} & 0.992 & $4.57372 e+9$ & 0.992 \\
\hline 5 & {$[2.16,2.27]$} & 0.985 & $4.53952 \mathrm{e}+9$ & 0.985 \\
\hline 6 & {$[2.29 \ldots 2.43]$} & 1.037 & $4.779200+9$ & 1.037 \\
\hline 7 & {$[2,44,2,75]$} & 3.048 & $1.40393 \mathrm{e}+10$ & 3.048 \\
\hline 8 & {$[2.88,3.08]$} & 1.008 & $4,64497 e+9$ & 1.008 \\
\hline 9 & {$[3.24 \ldots 3.45]$} & 1.792 & $8.25823 e+9$ & 1.792 \\
\hline 10 & {$[4.62 \ldots 5.16]$} & 2.178 & $1.00394 e+10$ & 2.178 \\
\hline 11 & {$[7,18,7,71]$} & 1.009 & $4.65046 \mathrm{e}+9$ & 1.009 \\
\hline 12 & {$[7,31,7,47]$} & 1.000 & $4.609566+9$ & 1.000 \\
\hline 13 & {$[7.62 .7 .92]$} & 1.014 & $4.67510 \mathrm{e}+9$ & 1.014 \\
\hline
\end{tabular}




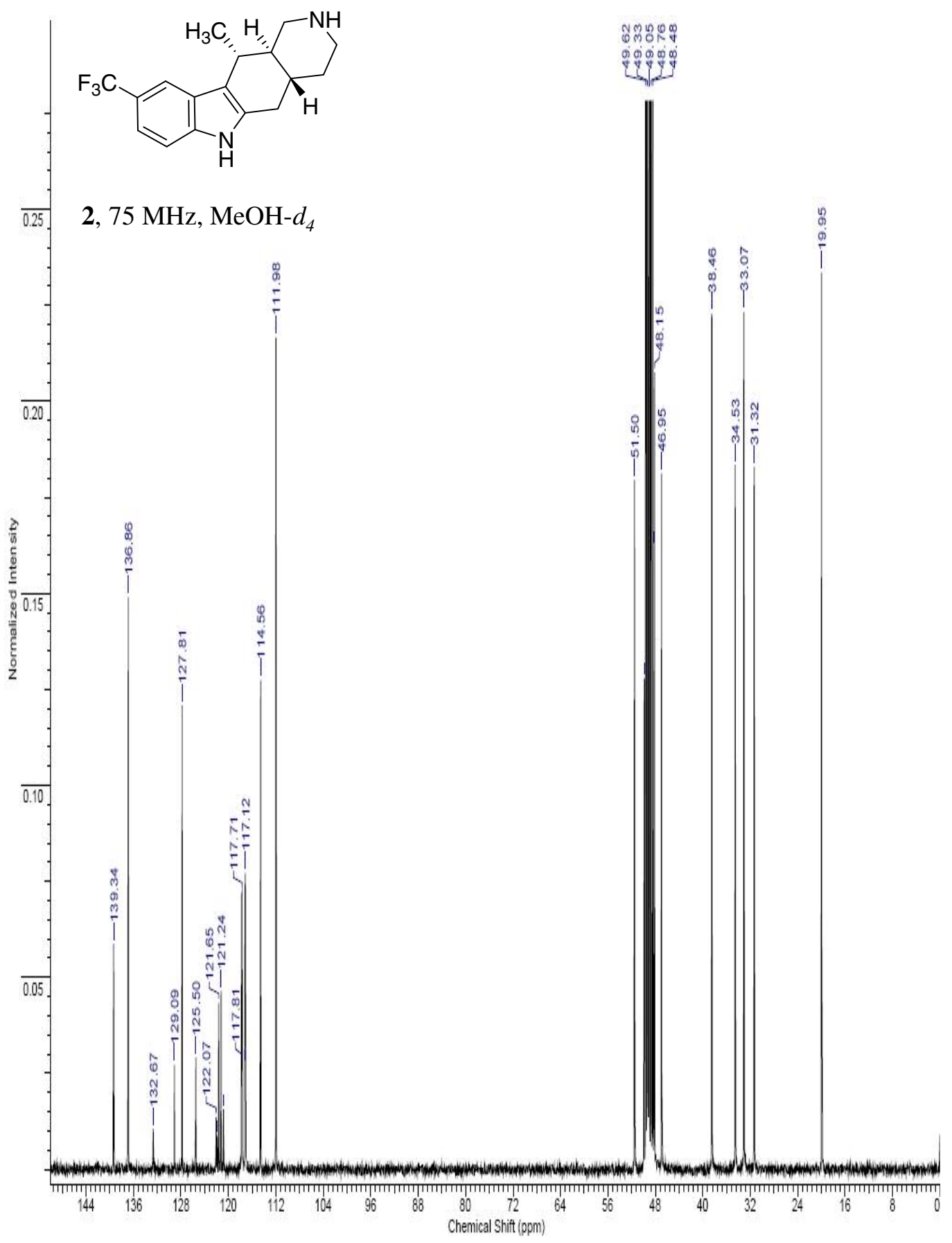




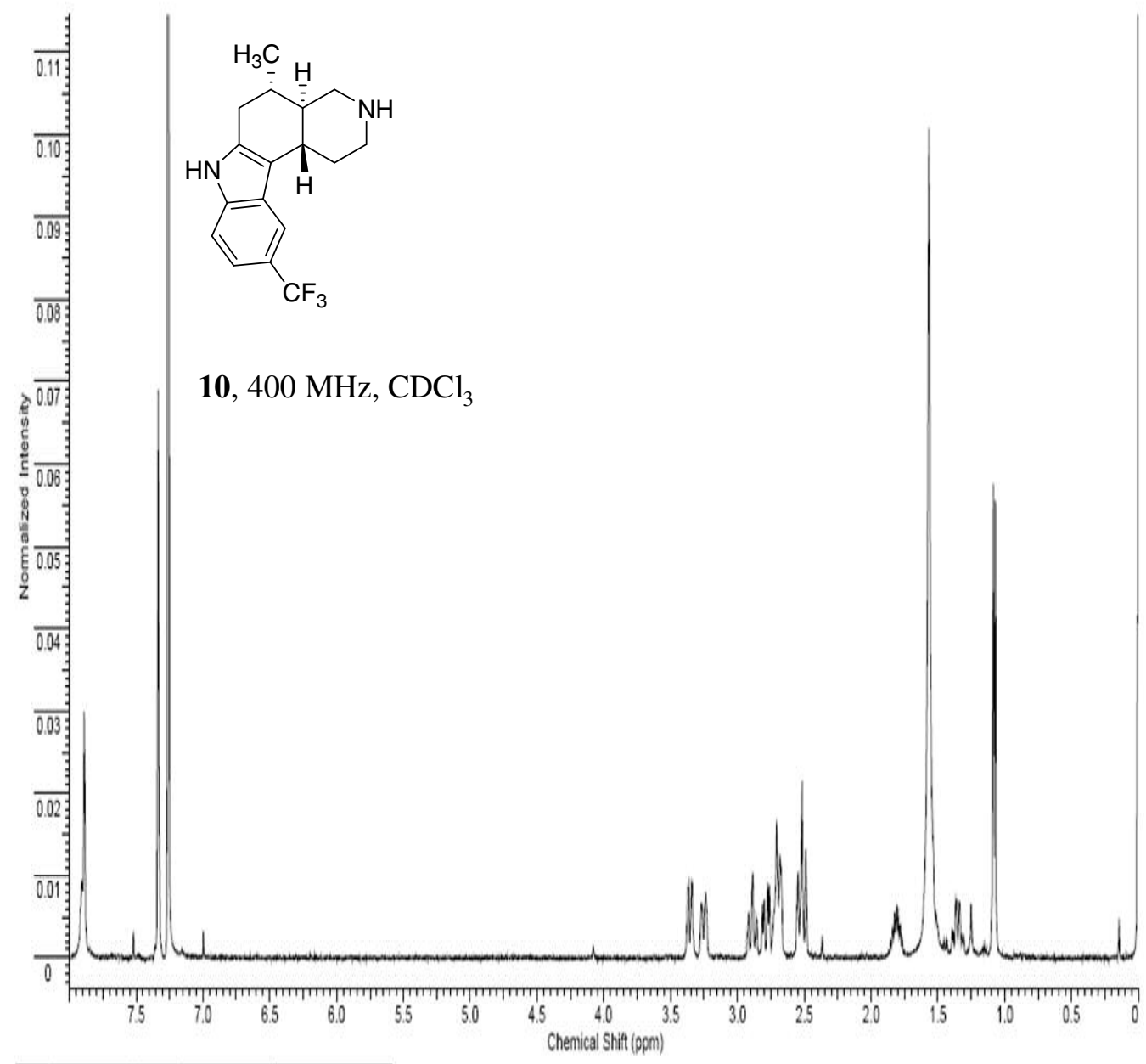

\begin{tabular}{|c|c|c|c|c|}
\hline №. & (ppm) & Value & Absolute Value & Non-Negative Value \\
\hline 1 & {$\left[\begin{array}{lll}1.01 & 1 & 1.12]\end{array}\right.$} & 3.000 & $7.11337 \mathrm{e}+8$ & 3.000 \\
\hline 2 & {$[1,29,1,42]$} & 1.181 & $2.80109 e+8$ & 1.181 \\
\hline 3 & {$[1,73,1,88]$} & 1.084 & $2.56981 \mathrm{et} 8$ & 1.024 \\
\hline 4 & {$[2.45,2.58]$} & 2029 & $4.81001 \mathrm{e}+8$ & 2.029 \\
\hline 5 & {$[2,63,2.74]$} & 2068 & $4.90447 \mathrm{e}+8$ & 2.068 \\
\hline 6 & {$[2,74,2.84]$} & 1.059 & $2.51138+8$ & 1.059 \\
\hline 7 & {$[2.84 \ldots 2.94]$} & 1.003 & $2.37782 e+8$ & 1.003 \\
\hline 8 & {$[3.26,3,30]$} & 0.389 & $9.21570 \mathrm{e}+7$ & 0.389 \\
\hline 9 & {$[3,30,3,43]$} & 1.086 & $2.57591 \mathrm{te}+8$ & 1.086 \\
\hline 10 & {$[7.28,7.41]$} & 1.988 & $4.71404 e+8$ & 1.988 \\
\hline 11 & {$[7,78,8.00]$} & 1.971 & $4,67430 \mathrm{e}+8$ & 1.971 \\
\hline
\end{tabular}




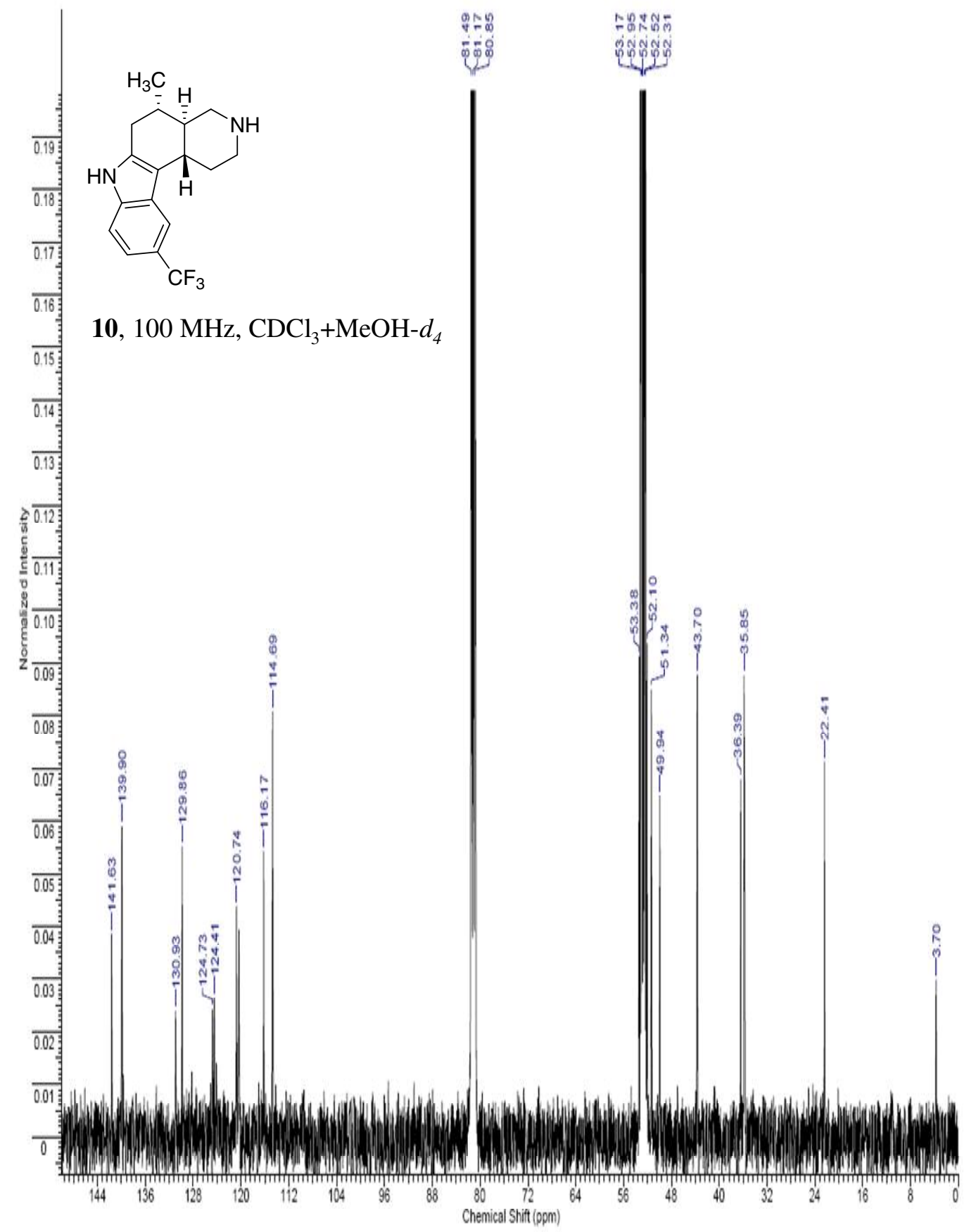




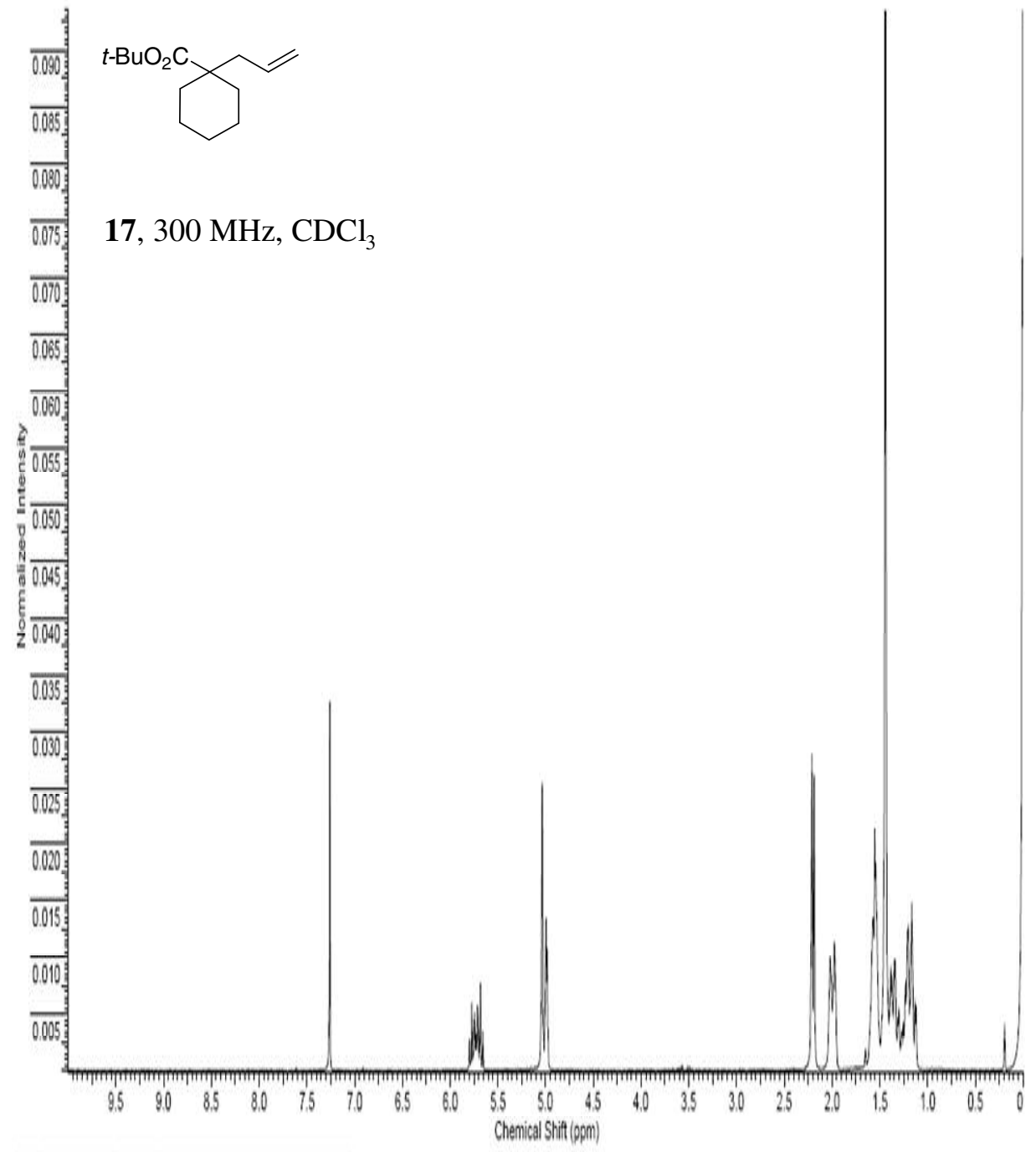

\begin{tabular}{|c|c|c|c|c|}
\hline No. & (popm) & Vave & Absolute Value & Non-Negative Value \\
\hline 1 & {$[1.08 \ldots 1,400]$} & 5.121 & $1.39484+6+9$ & 5.121 \\
\hline 2 & $|1,44 \ldots 1.51|$ & 7.264 & $1.978644+9$ & 7.264 \\
\hline 3 & {$[1.51 \ldots 1.728]$} & 3.367 & $9.172122+8$ & 3.367 \\
\hline 4 & {$[1.83 \ldots 2.11]$} & 2084 & $5.67534+6+8$ & 2024 \\
\hline 5 & {$[2.11 \ldots 2.33]$} & 2077 & $5.65742 \mathrm{e}+8$ & 20077 \\
\hline 6 & {$[4,84 \ldots 5,16]$} & 2038 & $5.550546+8$ & 2038 \\
\hline 1 & {$[5.55,5.81]$} & 1.000 & $2.723936+8$ & 1,000 \\
\hline
\end{tabular}




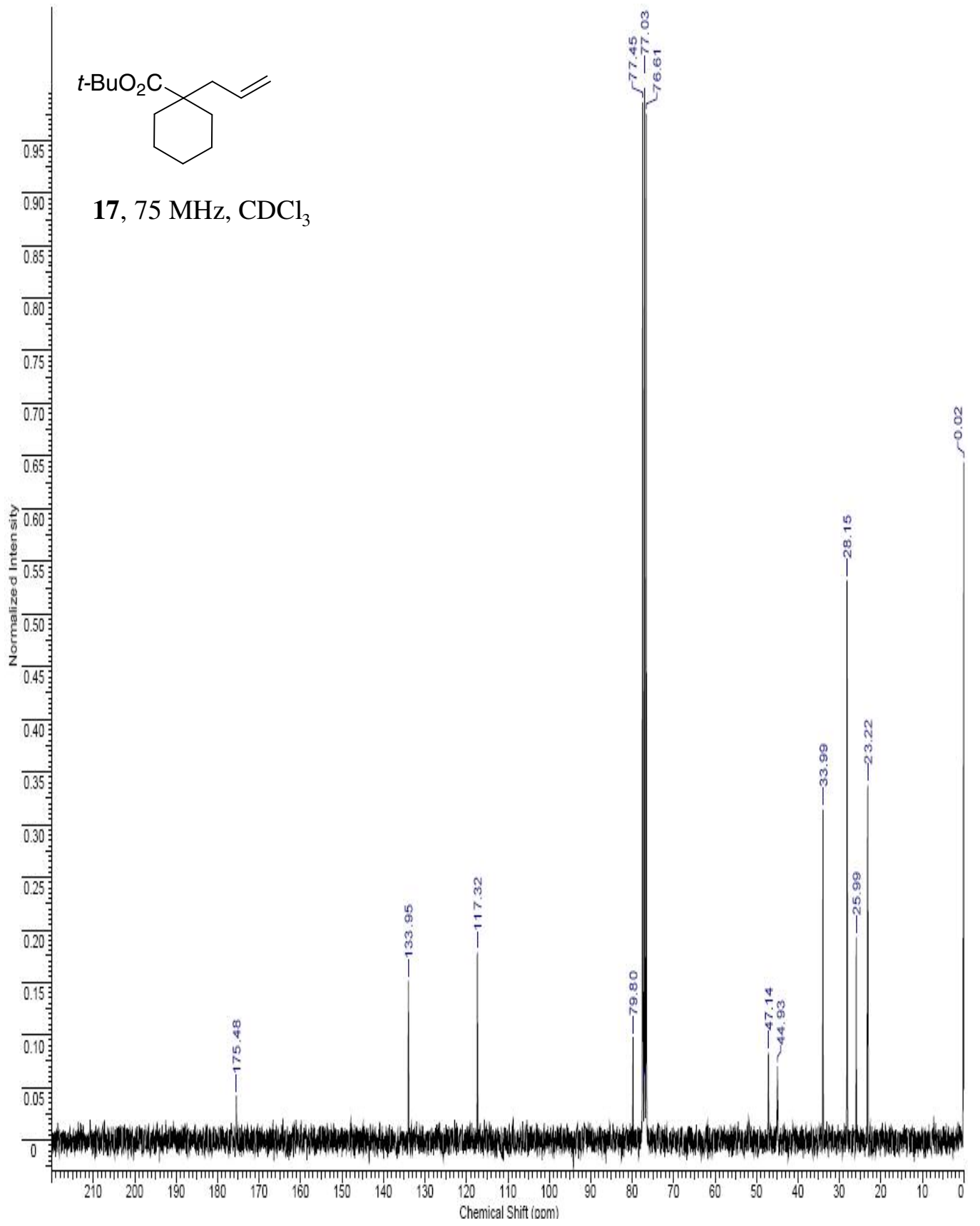

S-14 


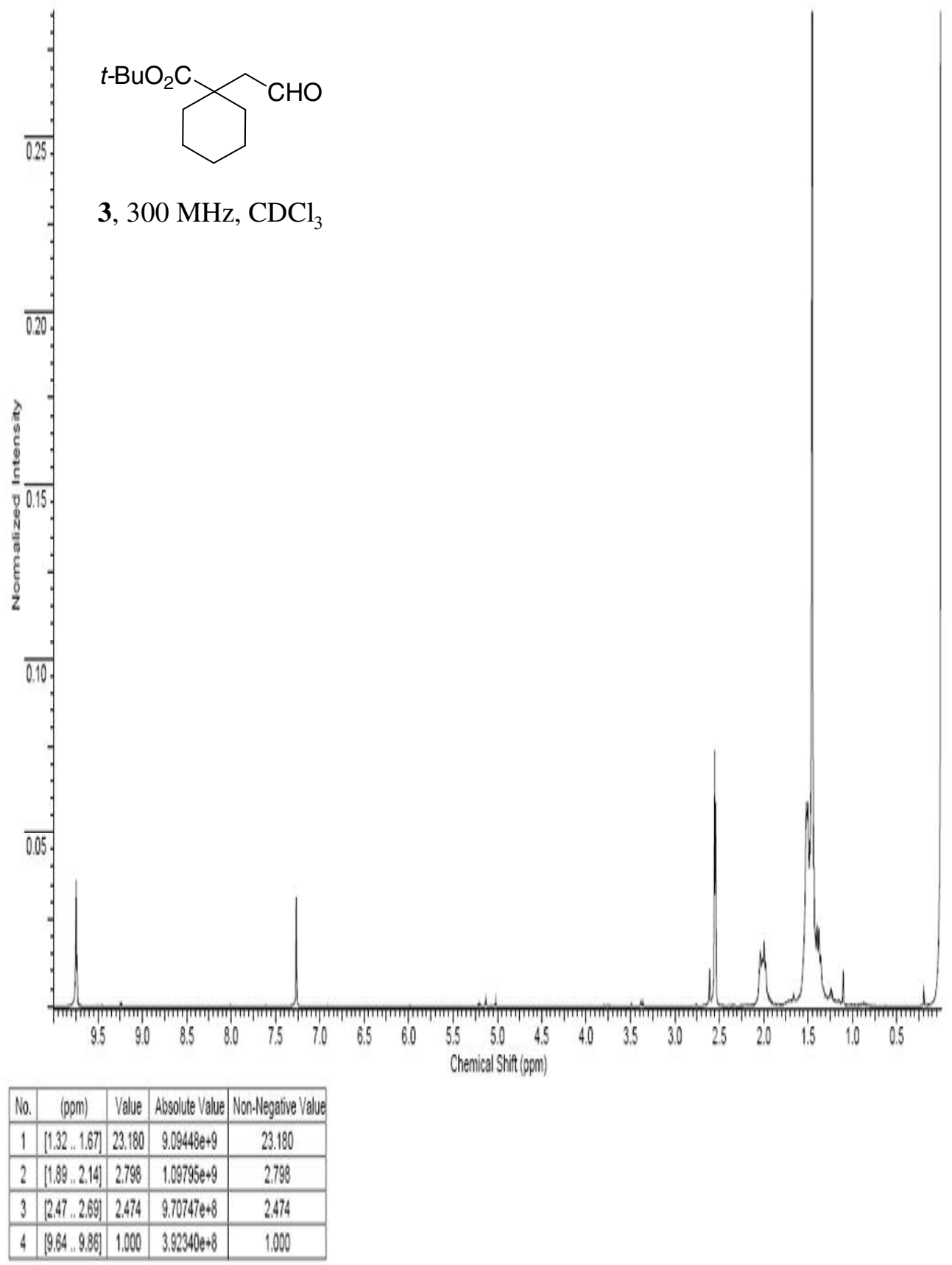

S-15 


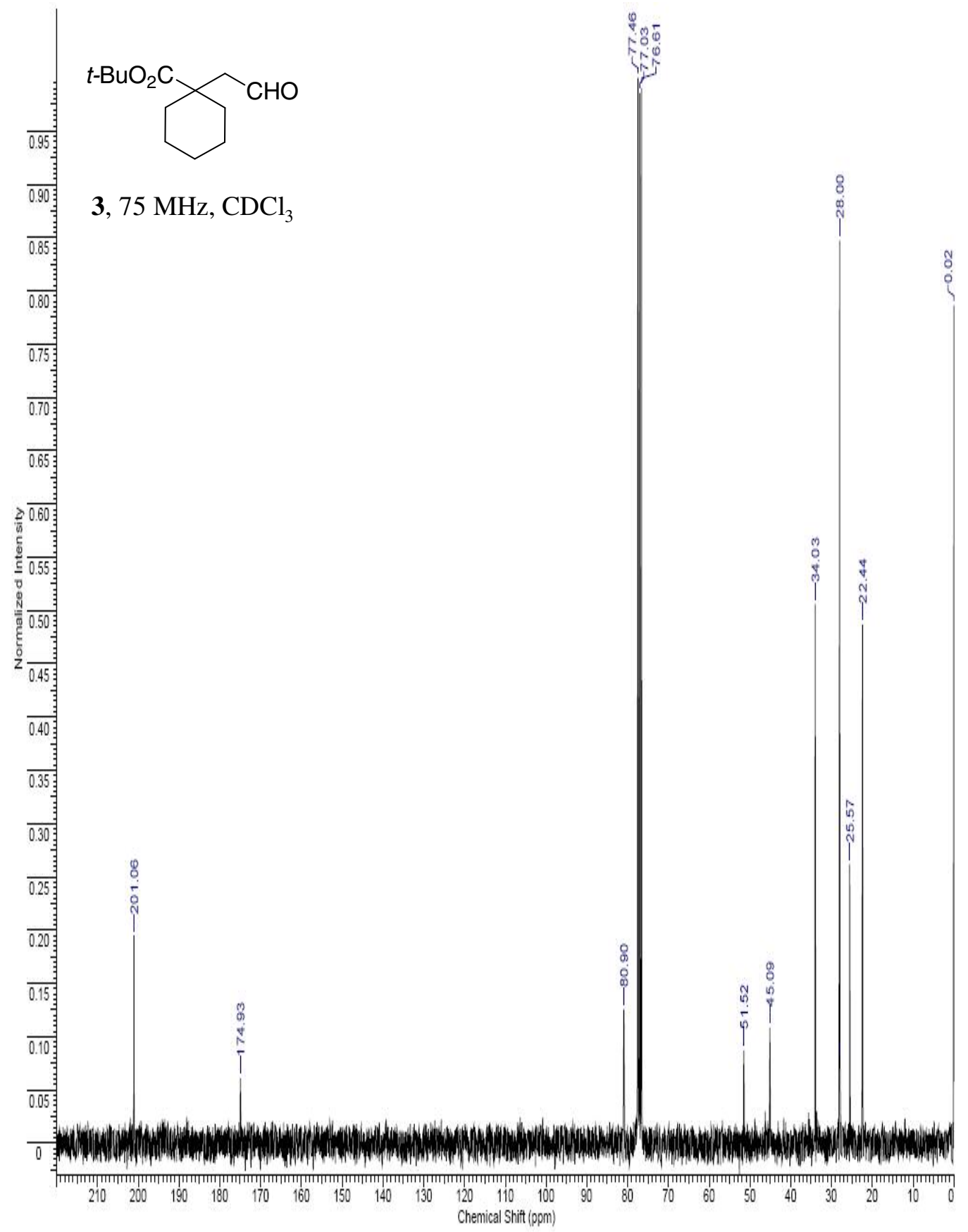

S-16 


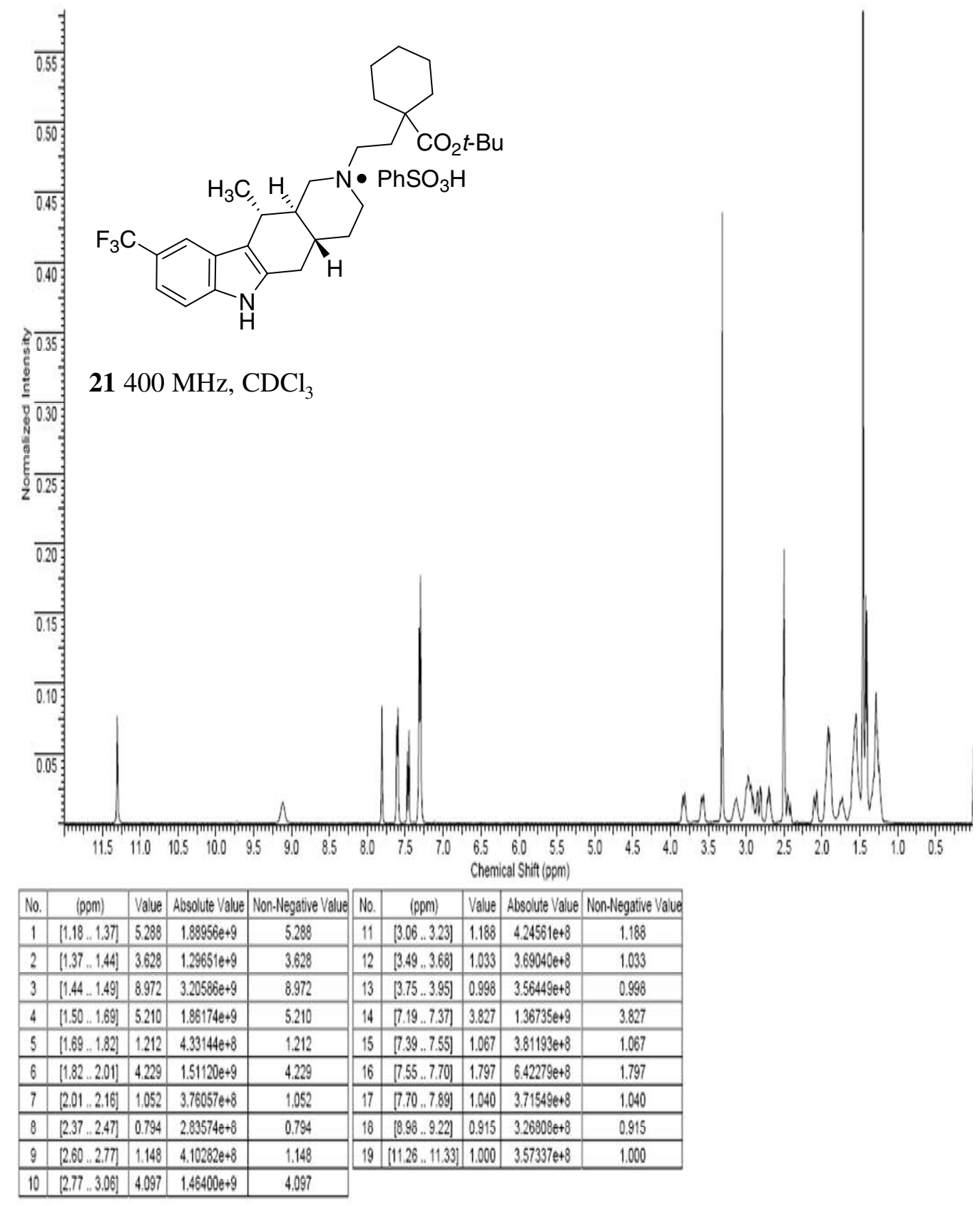




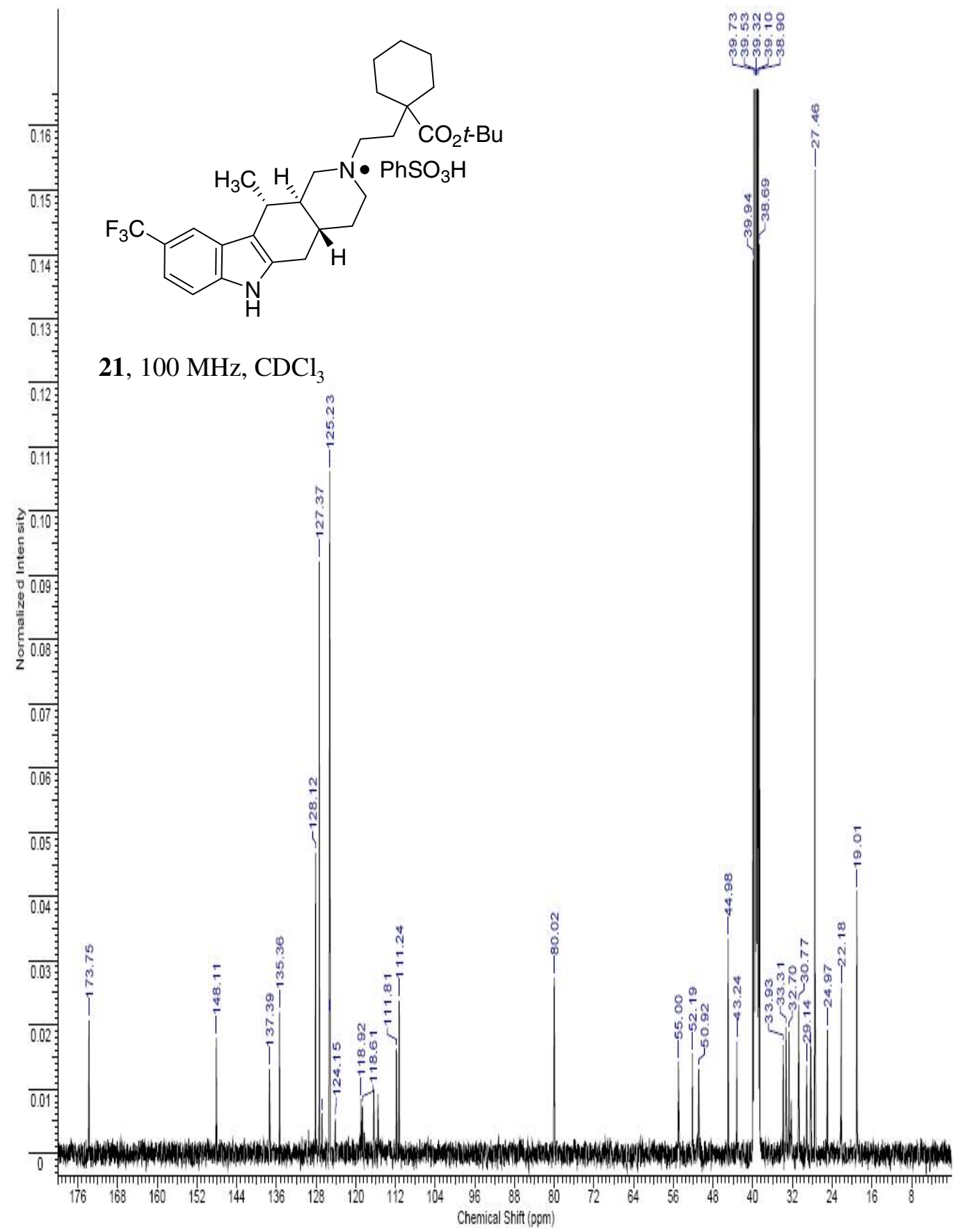




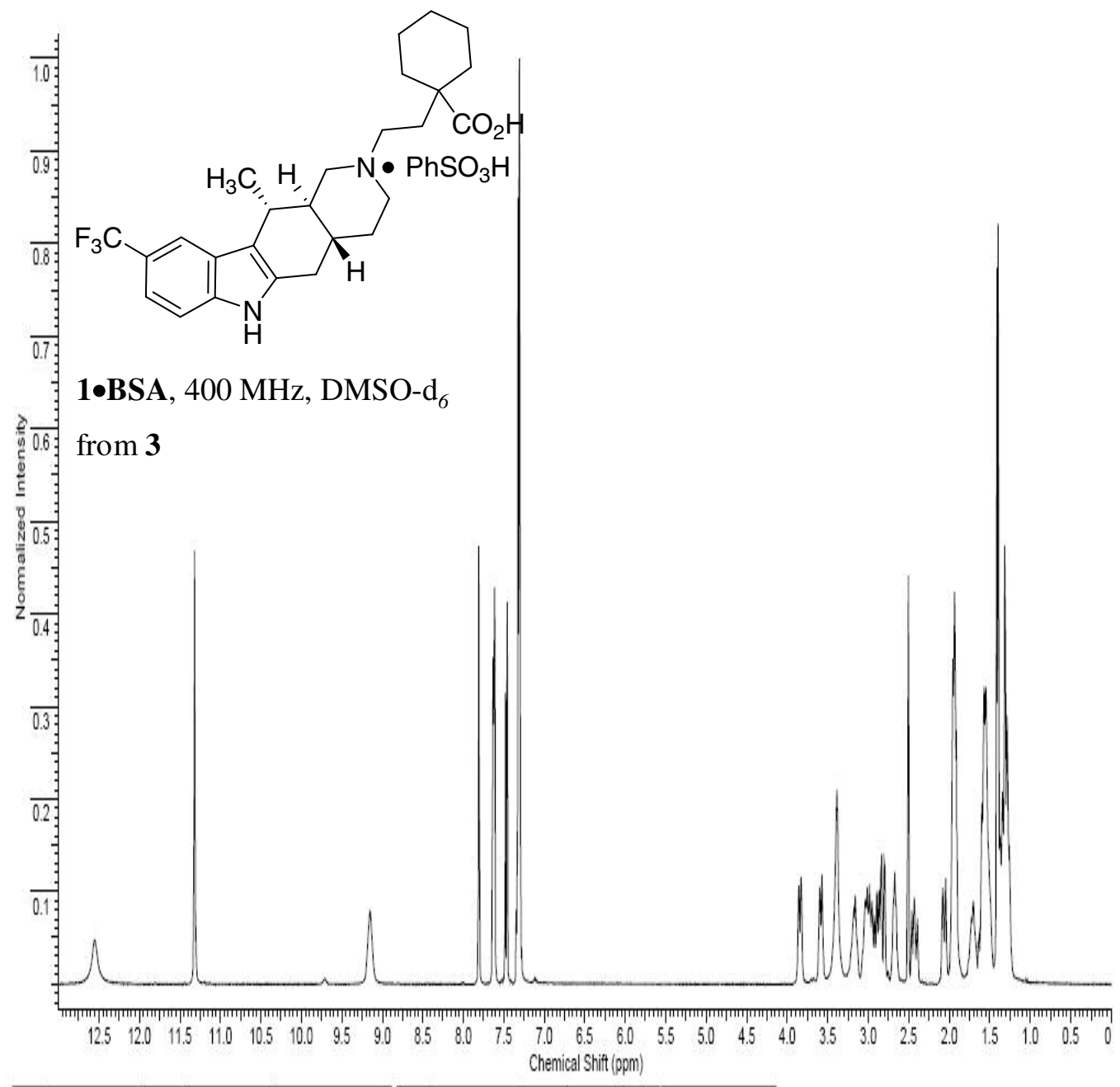

\begin{tabular}{|c|c|c|c|c|c|c|c|c|c|}
\hline №. & (ppm) & Value & Absolute Value & Non-Negative Value & No. & (ppm) & Value & Absolute Value & Non-Negative Value \\
\hline 1 & [1.10..1.46] & 8.765 & $4.40797 e+10$ & 8.765 & 11 & [3.51 ..3.65] & 1.040 & $5.22850 \mathrm{e}+9$ & 1.040 \\
\hline 2 & {$[1.46 . .1 .66]$} & 5.264 & $2.64727 e+10$ & 5.264 & 12 & [3.75 ..3.95] & 0.994 & $5.00044 e+9$ & 0.994 \\
\hline 3 & {$[1.66 .1 .80]$} & 1.157 & $5.81792 e+9$ & 1.157 & 13 & {$[7.18 . .7 .40]$} & 4.294 & $2.15977 \mathrm{e}+10$ & 4.294 \\
\hline 4 & {$[1.80 . .201]$} & 4.293 & $2.15906 \mathrm{e}+10$ & 4.293 & 14 & {$[7.40 . .7 .55]$} & 1.102 & $5.54002 e+9$ & 1.102 \\
\hline 5 & {$[2.01 \ldots 2.18]$} & 1.035 & $5.20392 e+9$ & 1.035 & 15 & [7.55..7.71] & 2.096 & $1.05433 e+10$ & 2.096 \\
\hline 6 & {$[2.29 .2 .54]$} & 2.139 & $1.07565 e+10$ & 2.139 & 16 & {$[7.74 \ldots .7 .85]$} & 1.056 & $5.30943 \mathrm{e}+9$ & 1.056 \\
\hline 7 & {$[2.55 \ldots 2.74]$} & 1.137 & $5.71829 e+9$ & 1.137 & 17 & [8.95 .. 9.33] & 1.000 & $5.02929 \mathrm{e}+9$ & 1.000 \\
\hline 8 & [2.74 . 3.09] & 4.167 & $2.09583 \mathrm{e}+10$ & 4.167 & 18 & [11.13.. 11.45] & 1.067 & $5.36739 \mathrm{e}+9$ & 1.067 \\
\hline 9 & {$[3.09 . .3 .27]$} & 1.224 & $6.15607 \mathrm{e}+9$ & 1.224 & 19 & [12.12..12.90] & 0.983 & $4.94455 \mathrm{e}+9$ & 0.983 \\
\hline 10 & [3.27 . 3.51] & 2.258 & 1.13563e+10 & 2.258 & & & & & \\
\hline
\end{tabular}




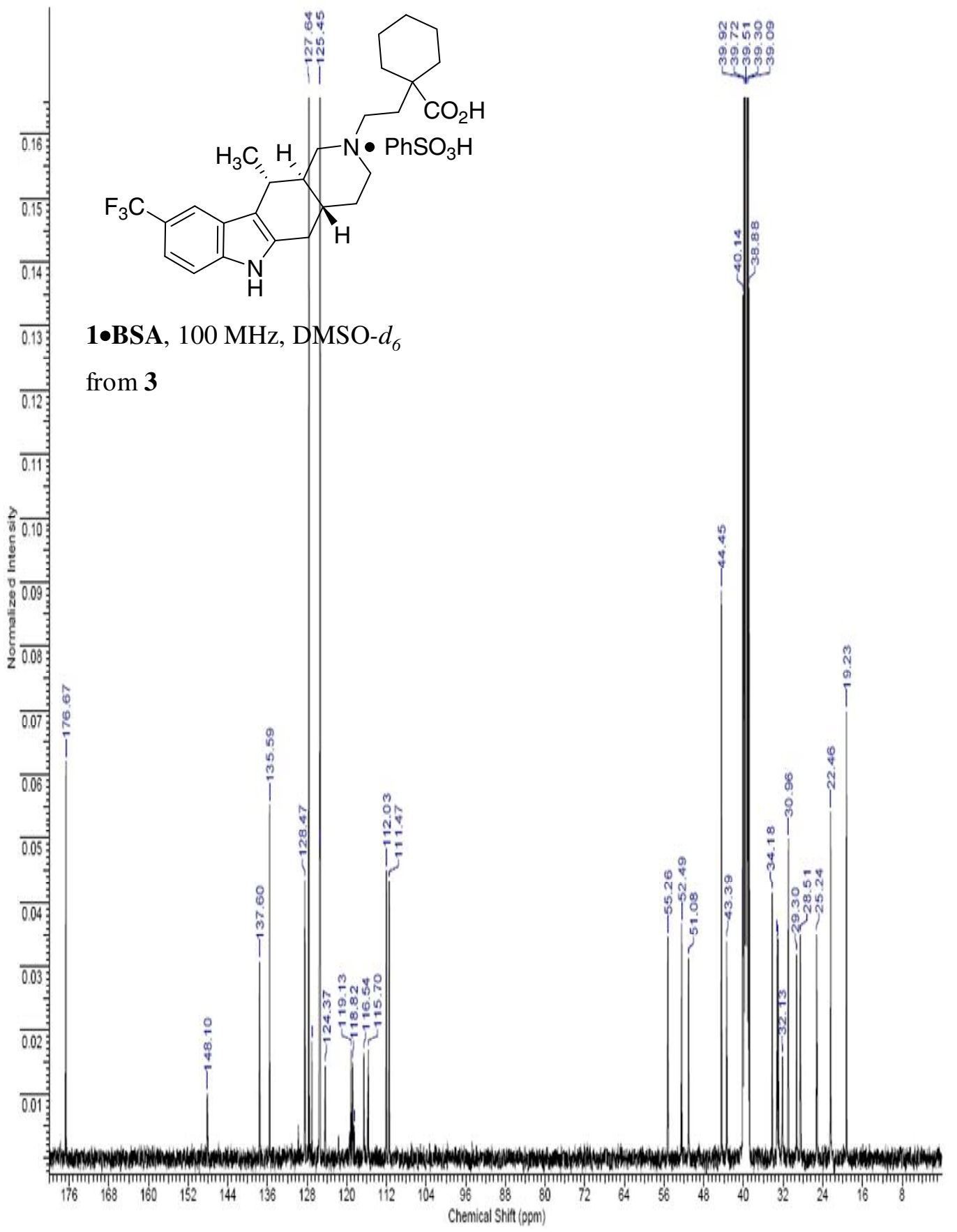




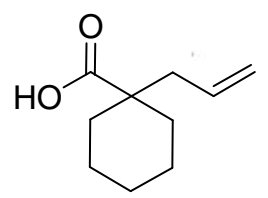

24, $400 \mathrm{MHz}$, DMSO- $d_{6}$

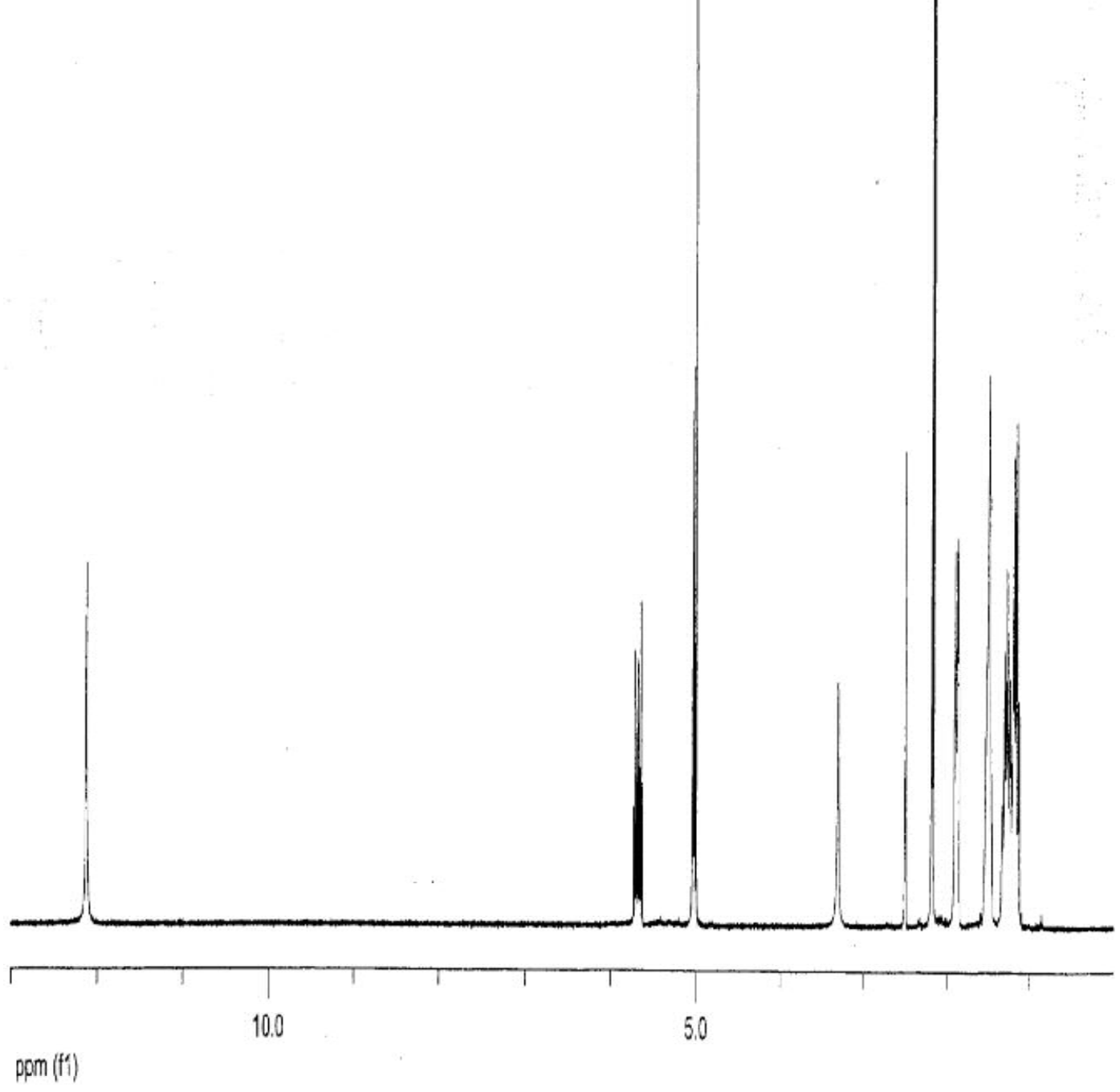




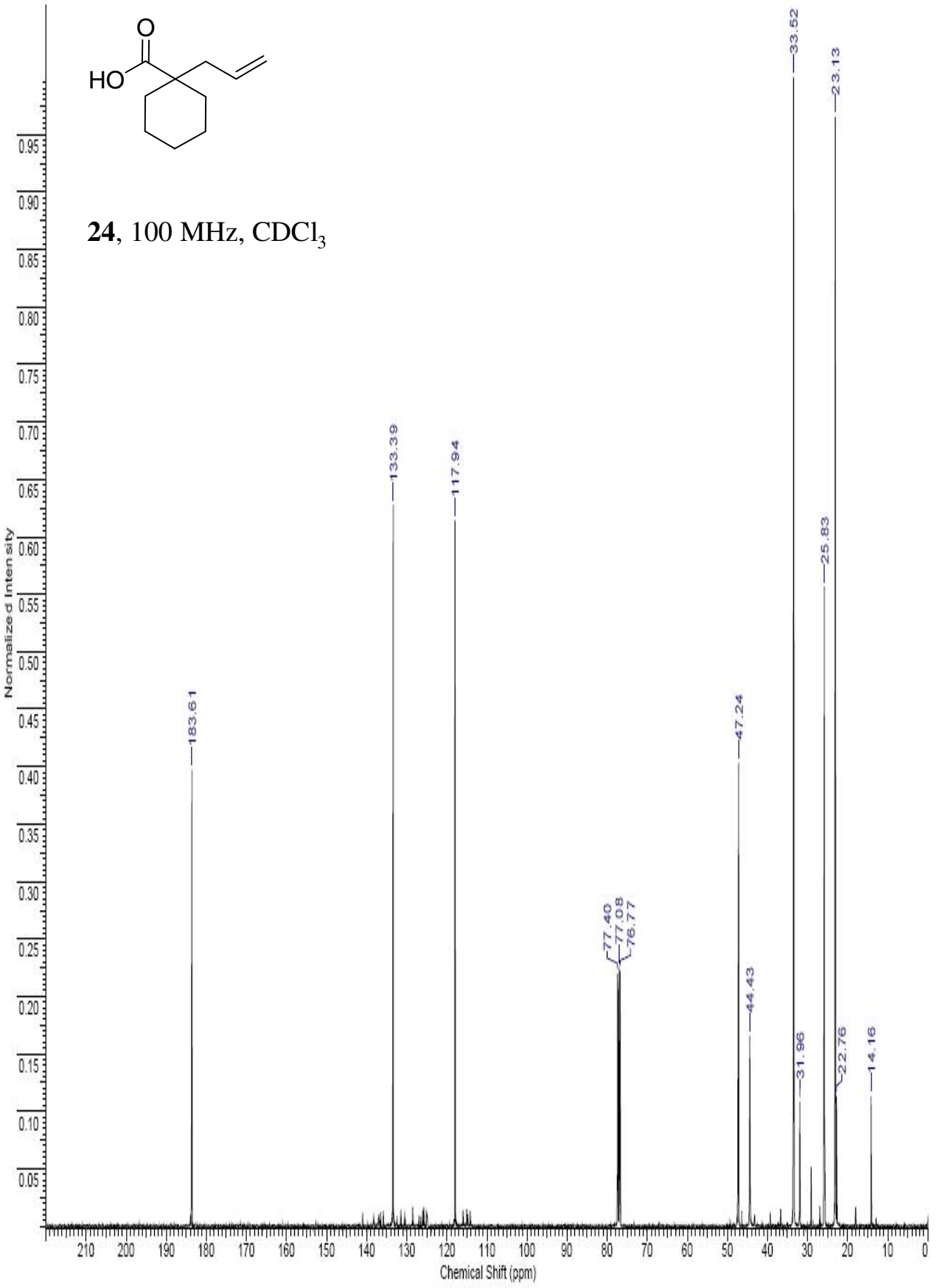

S-22 


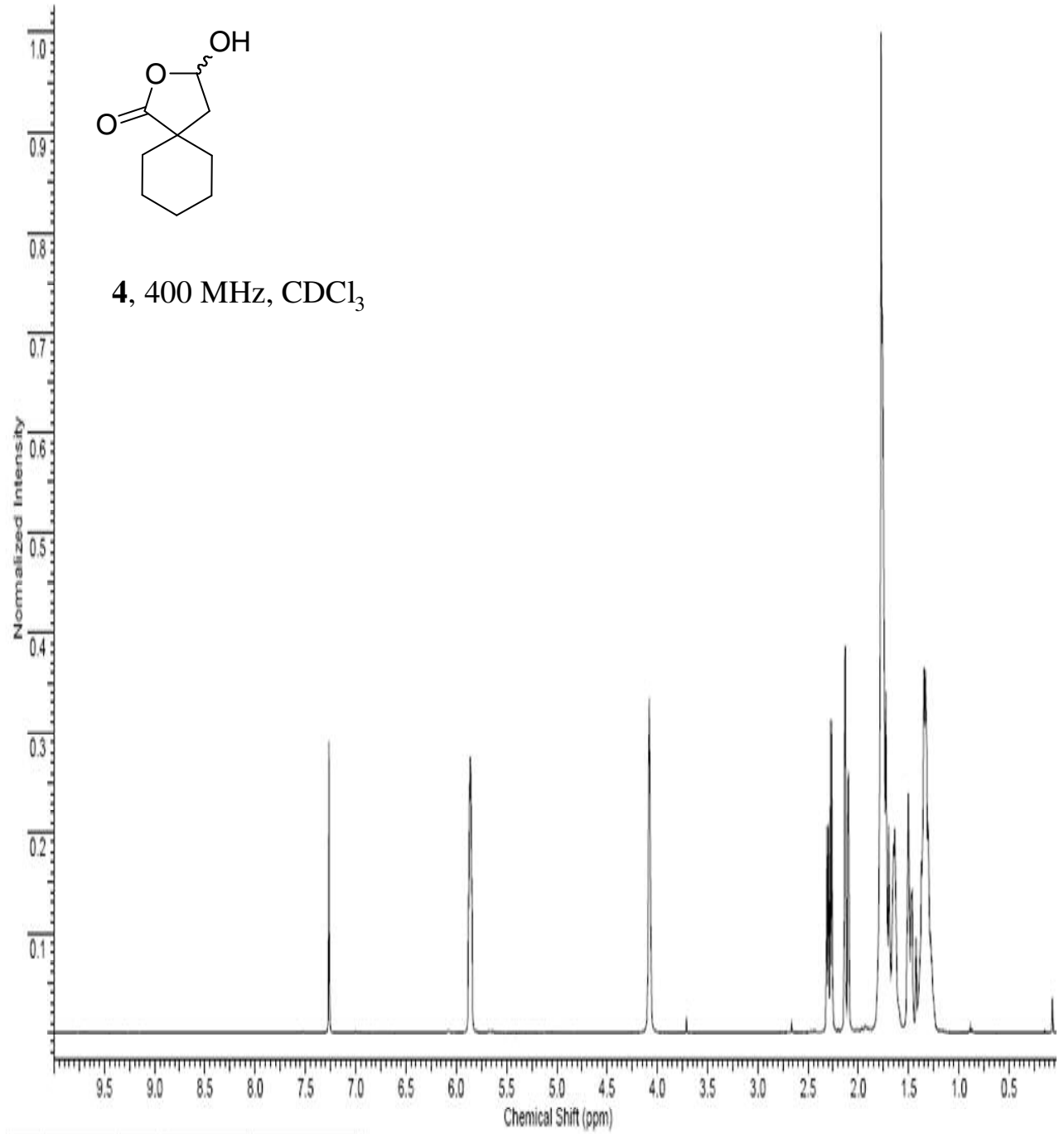

\begin{tabular}{|c|c|c|c|c|}
\hline & (pom) & Value & Absolute value & Non-Negative \\
\hline & $1.20,1.54]$ & 4.448 & $2.282399 e+10$ & 4.448 \\
\hline & {$[1.58 . .1 .87]$} & 6.436 & $3.303880+10$ & .4. \\
\hline & {$[2.03 \ldots 2.18]$} & 1.089 & $5.4866178+9$ & 106 \\
\hline & (22.20..238) & 1.069 & $5.499626+9$ & 1.069 \\
\hline & (3.988.4.422. & 0.985 & 5.054933e+9 & 0.985 \\
\hline & $\mid 5.76,5999]$ & 1.000 & $5.13318 \mathrm{eet}$ & 1.000 \\
\hline
\end{tabular}




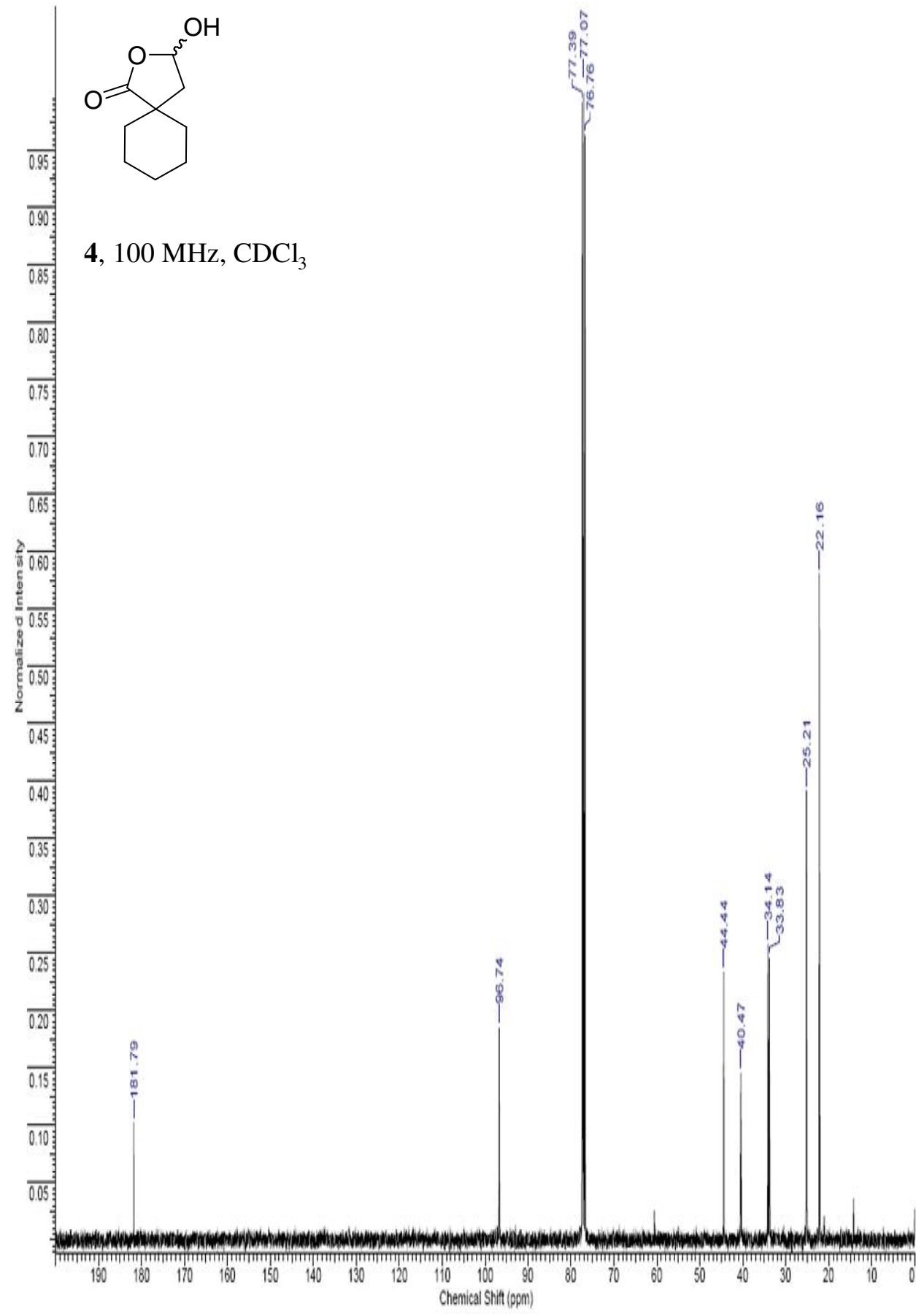

S-24 


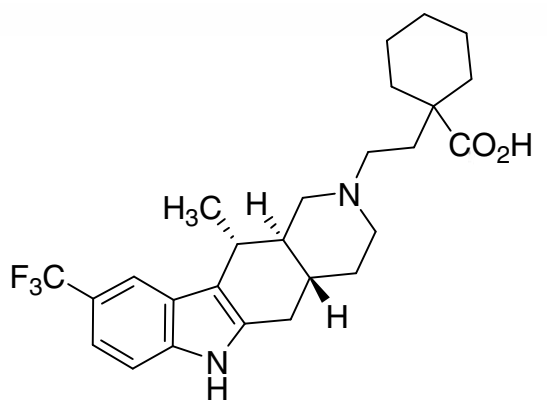

1, $600 \mathrm{MHz}$, TFA- $d_{1}+$ DMSO- $d_{6}$ from 4

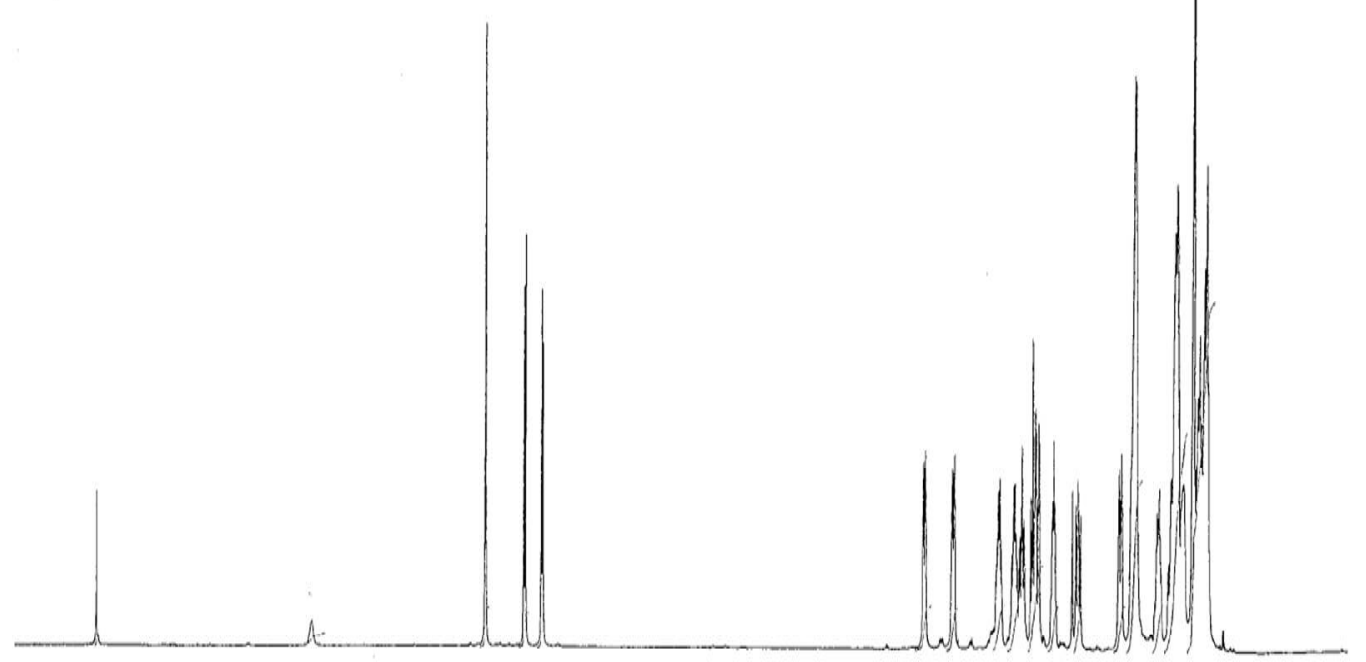

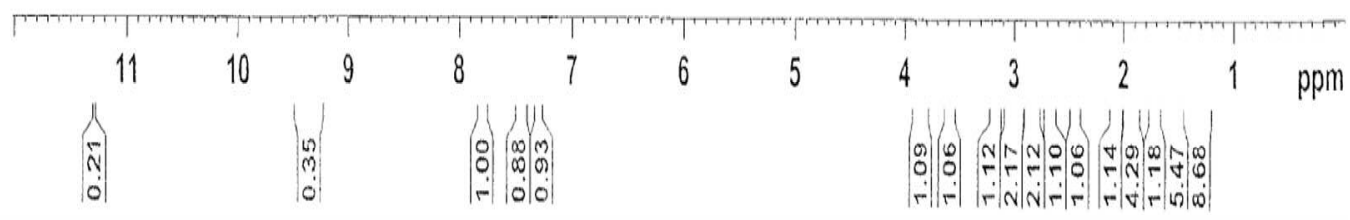



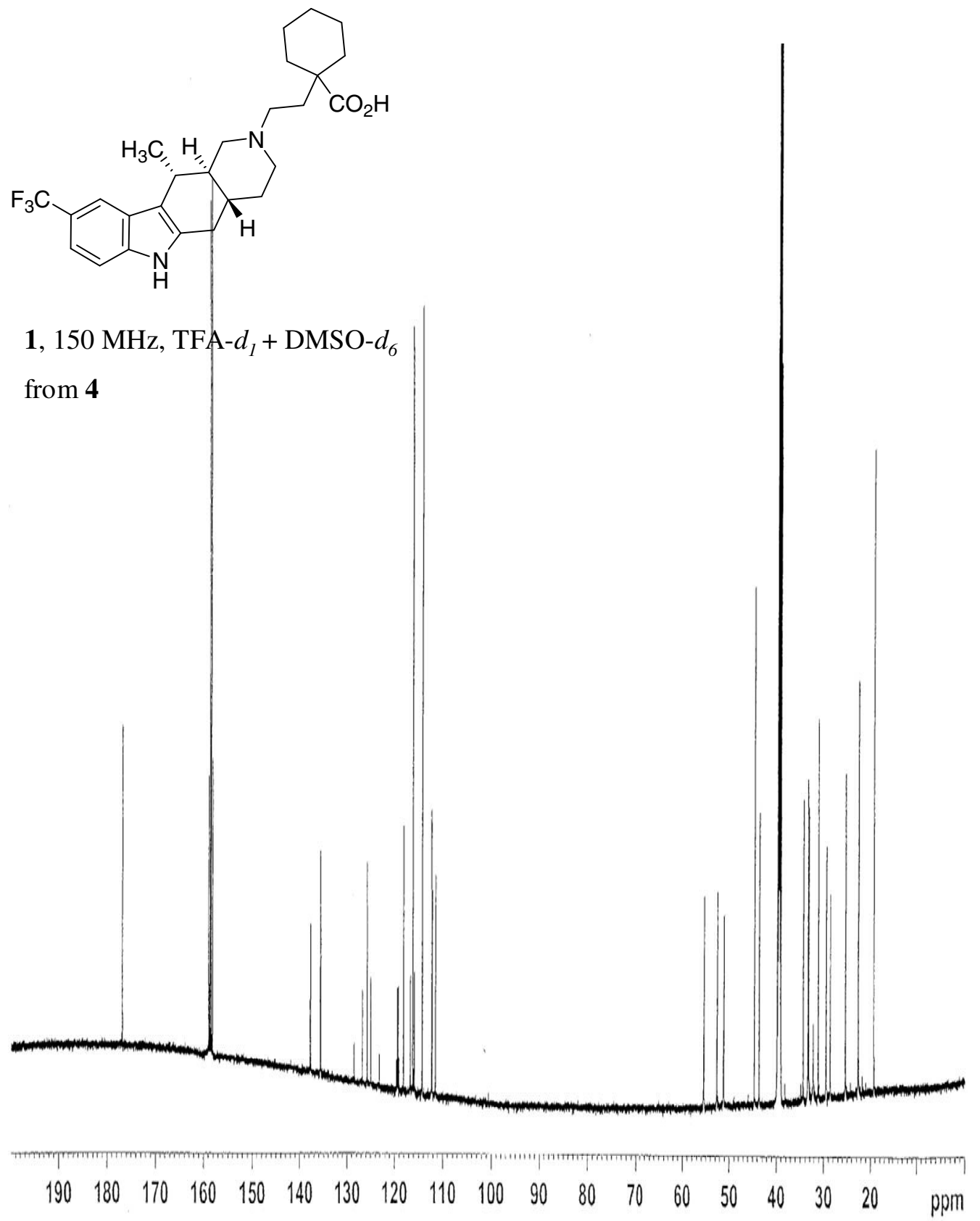
Section C: ORTEP $(R)$-2-Chloromandelate salt of 2

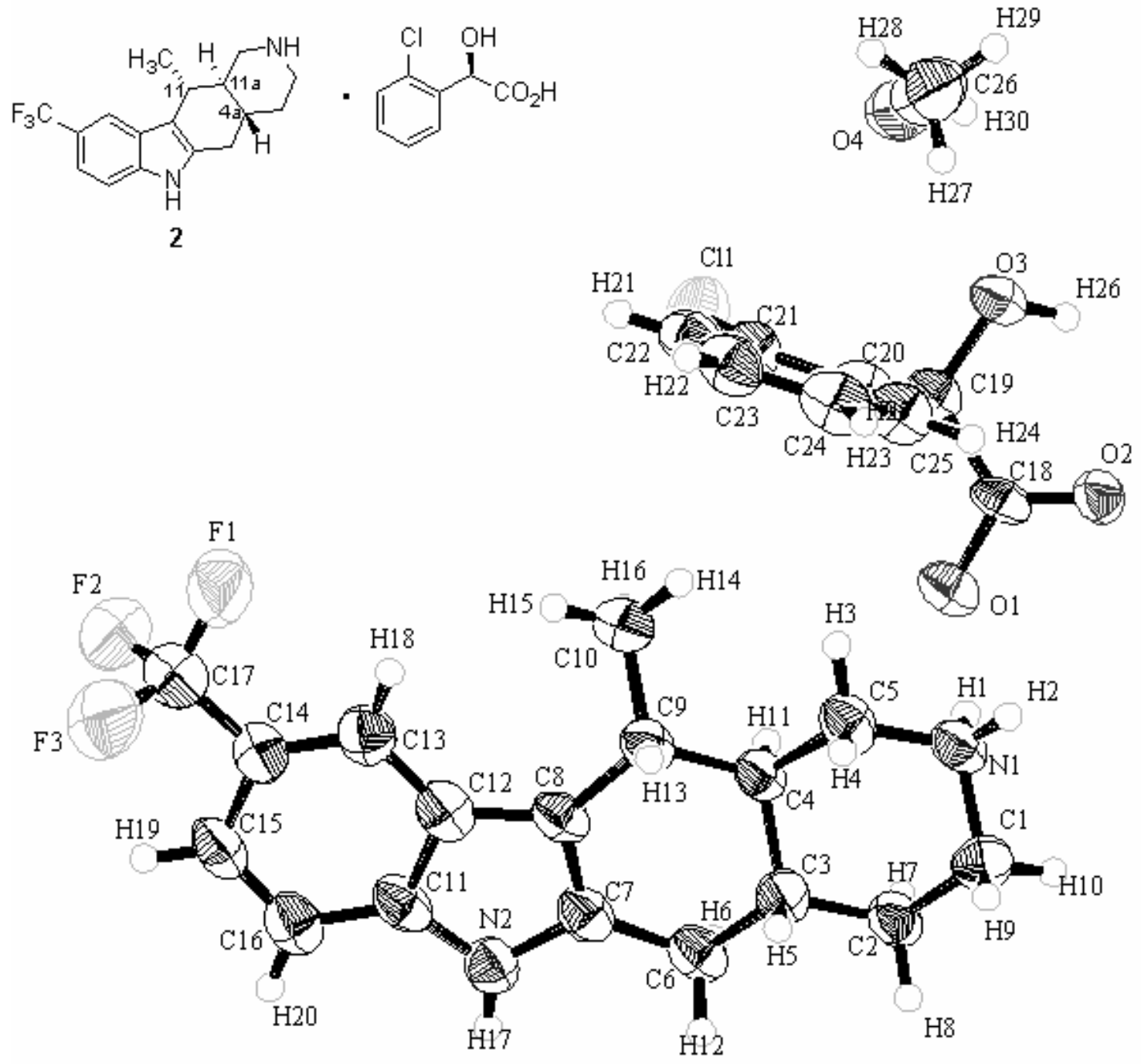


S-28 